\title{
Food behavior as risk factor for developing eating disorders
}

\author{
Walter Milano ${ }^{1}$ and Anna Capasso ${ }^{2 *}$ \\ ${ }^{1}$ Mental Health Unit, District 24 ASL Napoli 1 Center, Italy \\ ${ }^{2}$ Department of Pharmacy, University of Salerno, Salerno, Italy
}

\begin{abstract}
The present review was focused on the Eating Disorders (ED), the role of neurotransmitters and peptides involved as well as the drugs used in the treatment of these diseases. For ED we intend a syndrome characterized by an persistent alteration of eating behavior and the conducts that cause an insufficient ingestion and/ or adsorption of aliments. We distinguish three different diseases in the ED: Anorexia Nervosa (AN), Bulimia Nervosa (BN) and Binge Eating Disorders (BED). The ED are complex conditions that arise from a combination of long-standing behavioral, emotional, psychological, interpersonal, and social factors. The neuronal circuits that control the ingestion of food is mainly related to catecholaminergic, serotoninergic and peptidergic systems. In this respect, while serotonin, dopamine and prostaglandin promote the ingestion of food, on the other side. neuropeptide $Y$, nor-epinephrine, GABA, opioid peptides inhibit it thus causing the insurgence of ED. The drugs mainly used in the treatment of ED are the antidepressants as the selective serotonin reuptake inhibitors and tricyclic antidepressant. Also, mood stabilizers (lithium), anxiolytics, serotonin and noradrenalin reuptake inhibitors and antipsychotic are often used in the treatment of ED.
\end{abstract}

\section{Introduction}

With the term alimentation we intend the satisfaction of psychological need of the hunger and of the thirst of which has necessity the organism to survive. This necessity is physiological, not psychological but a condition of physiological necessity has psychological consequences that assume the name of "drive" [1-5].

About the alimentation it was told all and the contrary of all: several fields are involved like the medicine, the chemical, the biology, the psychology, the history, the anthropology, the sociology and the list could extend until to include all of disciplines of the human knowledge.

How unexpected senses are hidden back alimentary behaviours [1-5].

We think to be free in the choice of that we eat but instead it is not so: we are bridled by a series of innumerable factors of which we usually are not conscious: the culture, the religion, the tradition of place where we live, only for to cite some examples. Religious norms have a superior power on the collective imaginary, cultural norms, instead, are a phenomenon that acts to level more superficial; it is a phenomenon that has a more strictly sociological importance.

To eat, to feed oneself, as we have saw, has numerous valences. As human beings we have an anatomic structure capable to digest and then to absorb almost all: by the secretion to go rancid of the mammary glands to mycetes, to the rocks crags or rather cheese, mushrooms and salt if you prefer the euphemism. But there are also much substance that the human beings have fair to eat even if perfectly edible by a biological point of view. This is proved by the point that in certain place some social group eat, finding delicious what other groups despise or detest. The food should nourish the collective mentality before can come in an empty stomach. Or rather there are food "good to think" or food "bad to think" [1-5].

We cannot forget that food is the "symbolic" instrument for excellence and that performs a role of identification in a group and therefore of delimitation of this group. This needs to delimit limits between social classes, geographic regions, cultures, sexual race, the cycle of the life and to distinguish, rites, traditions, feast, seasons, ext.
But the symbolic function of feed oneself is no high, particularly in the child, that, as saw Freud, the erotic and inter-relational component prevails on the biological sense. Around the nutritive function is organized all of psychological world of child, his feels, the way of reporting with the external world, his anxiety and fear, the modality to express pain and aggressiveness. With the alimentation, the new born expresses also an erotic sense. This connotation is connected to the first form of construction of his identify and his knowledge of the other; to the pleasure caused by the stimulation of the mouth, typical of the oral phase, to which, in the following evolutive process, the individual can remain fixed or can regress [1-5].

\section{Alteration of eating disorders}

This short digression on values given to alimentation by the human race has served to introduce the part that interests to the most medicine, psychology and clinical, that is the alteration of eating disorders. For Eating Disorders (ED) we intend a syndrome characterized by an persistent alteration of alimentary behaviours and conducts connected with the food that cause an insufficient ingestion and/ or adsorption of aliments. This disease, caused not directly by internist disease or by other physical disease, can compromise in a serious way the psychosocial functioning and the physical welfare of patient.

These disease are phenomenon frequently discovered in the clinical practice and under the name of ED, they are included 3 clinical picture of psychiatric and internist interest: Anorexia Nervosa (AN), Bulimia Nervosa (BN) and Binge Eating Disorders (BED) [6-10].

In the occidental culture the first clinical descriptions of alimentary anomalies or similar conditions to those today defined ED date back to over 2000 years ago.

Correspondence to: Anna Capasso, Department of Pharmacy, University of Salerno, Salerno, Italy, E-mail: annacap@unisa.it

Received: November 06, 2017; Accepted: November 28, 2017; Published: November 30, 2017 
Reconstructions of the life of some Saints represent a clear example of anorexic behaviours. The aspects of hunger and of insatiable voracity are also present in the ancient Greek and Hebraic literature, such as typical and caricatural figures of the obese, of their irrepressible greediness and gluttony [1-10].

Even if know since antiquity, only recently these disorders have been object of systematic researches and studies on the psychopathological, neuroendocrinological and clinical plane; considered for a long time endocrinological or internist diseases or symptoms and variants of other nosographic sphere like hysteria, obsessive neurosis, schizophrenia or depression, the ED have today their nosographic connotation [1-10].

The ED are introduced in mental diseases and are included in the area of competence of the psychopathology, psychiatry and psychotherapy. But, in the mental disease, the ED are those that present most zones of intersection with other sectors of medicine and that must require, particularly in some phases, coordinate interventions of different specialists. In the occidental countries the prevalence of $\mathrm{BN}$ in the young is included between 1 and $3 \%$ and oscillates about to the $0,5 \%$ for anorexia nervosa. The $3-5 \%$ of females of the same age suffers partial symptoms. In adolescents the prevalence of anorexia nervosa is estimated of about $0,8 \%$ and the prevalence of bulimia nervosa about of $5,8 \%$; the partial syndromes implicate by 1,7 to $13,5 \%$ of population of the secondary schools. We can say that 8-10 females on 100 , aged between 12 and 25 (the age more to risk), suffer of some forms of ED. The mortality is important: it is included between the 5 and $18 \%$ for AN and $7 \%$ for BN [10-20].

A diagnosis of ED can be formulated when in the patient there are, at the same time, alimentary behaviours seriously disturbed associated to precise signs and symptoms. The alterations of alimentary behaviours can be identified directing the psychological investigation to followed levels of observation:

- Sense of hunger altered: the sense of hunger lose its significance of control on the caloric provisioning. The sensation is released by the central control, to bind it self more to intra-psychic stimulus.

- Control of impulses altered: the patient shows a difficulty to control his behavioural impulses that can pour also particularly on the control of alimentary stimulus.

- Alteration of somatic image: the subject lose the capacity to formulate an objective opinion on his body image. In the extreme cases, states of extreme thinness are defined like condition of overweight.

- Alteration of reality: the subject has difficulty to formulate an objective opinion on the surrounding reality. He is not able to structure a clear idea of demarcation between internal and external reality.

- Alteration of the somatic coenesthesis: it is the incapacity to distinguish the physiological sense of much coenesthesic perceptions.

- Coenaesthesia: immediate and general perception of own body, determinate by the totality of sensations, not always conscious, made possible by the presence of proprioceptors of internal systems. The consciousness of somatic oneself. The coenesthesia is the result of a complicated interaction between physical and emotional affective condition)

- Alteration of the relational control: the communication with others elements of his habitat is disturbed.
The fear to fatten remains the common and nuclear element of the different form of ED, in AN in BN and BED. The definition of ED not otherwise specified, allows to underline the importance to consider as entity clinically prominent also partial syndromes, that is some forms where the symptoms are present less numerous: these, present both in the population not clinical that in the population in therapy, can anticipate or follow a complete syndrome and are generally taken by a significant and clear psychological suffering (depression, attempt of suicide, somatoform diseases). The expression of a complete syndrome give back particularly important, in order to the secondary prevention, their individualization. Recent studies show a modification of the absorption of symptoms in the different syndrome: the symptoms bound to psychological dimension (fear to fatten and desire of thinness) are more frequently verified, while symptoms that interest the somatic sphere are in progressive reduction (loss of weight and amenorrhoea). The survey of a population less grave medical point of view could be the indication of an improvement of the potentiality of a precocious diagnosis [10-20].

The gravity of ED is one of indicator of the level of compromise of patient that will result useful at following indication to treatment. The gravity of this disease can be described and valued by a qualitative and quantitative point of view.

The quantitative evaluation (entity of loss of weight, frequency of bingeing and purgative behaviours) avails it self of the determination presence/absence of behaviours and their influence on the clinical parameters (BMI, hemato-chemical value, ext); the quantitative evaluation, instead, is related to polarization of thought on the ED: of will be determinate the extension and the possibility to modify this during of clinical colloquy [10-20].

It exists, in the attempt to know what can determine the evident increase of the diffusion of ED, a cultural problem. The diffusion of "dieting" to internal of all occidental population could induce to increase the tendency to assume restrictive alimentary styles and to put on outward appearance and identity. This explanation could induce arrange the ED like placed to the external "pathological" of a continuum of behaviours that starts in the "physiological" social or professional worry for the weight [10-20].

The strict interlacement between normal and pathological behaviours also allows that doctor (medical, psychiatric or psychology) had requests, formulate directly by the patient or by his familiars, in absence of a real clinical necessity. An other aspect that gives back complex the indication to treatment is the diagnostic competence. The ED are psychiatric diseases. Nevertheless their physical pathogenesis, or the use of body as means of expression of the suffering, has caused ambiguous in relation to the diagnostic and consequently therapeutic, of these diseases. Are developed diagnostic approaches directed within certain limits to the organic components of the disease (extreme organization) and vice versa approaches that dangerously don't consider the importance of (extreme psychology) [20-30].

In both cases the clinical risks to ratify and perpetuate the division between emotionality and corporality, at the origin of the disease for all patients, even though they used in different kind, that the clinical should to seize and to retrace to the object of care. The identification of dignity and importance to different ambits of competence will sometimes take to a contemporary engagement of clinics, psychiatrists, internists, psychologists, endocrinologists, or nutritionists. This engagement will be shared for a global and correct organization of patient. The possible use of a multidisciplinary team imposes to observation of different 
style of relation that patients can put in act with them, in relation to their different professional, personal characteristics and in relation to different places where the clinical meet the patient (private study, dayhospital, ambulatory or hospital) [20-30].

\section{Anorexia nervosa}

The term "anorexia", that means loss of appetite, is inappropriate. In fact, even though these people refuse the food, generally they preserve their appetite and, like most people, they think excessively to the food.

The illness generally start by the first at the late adolescence, interesting for the $95 \%$ of cases the female sex. The loss of weight is due to the drastic reduction of the taking daily of food, that frequently is followed by self-induced vomiting, abuse of laxatives, diuretics ext. This impressive loss of weight is followed by a series of somatic symptoms that are due to the consequences of the poor state of health variable, to time of extreme level. The principal characteristic of $\mathrm{AN}$ is the refusal to preserve the normal corporeal weight, that is correlated to the extreme fear to fatten. The latest edition of DSM IV of 1994 expounds the diagnostic criteria of AN, specifying besides the possibility to divide the patients affected by $\mathrm{AN}$ in 2 subgroup, with or without purging behavioural [20-30].

\section{Clinical picture}

Generally the premorbid characteristics are similar: timid, submissive, perfectionist and competitive children and adolescents; conscientious, aimed to obtain the maximum by all performance. The modality of beginning more frequent is that gradual and insidious: an adolescent really in overweight sometime, decides to begin a diet lose some kilo, tends progressively to depart herself by hers contemporary, often she makes on activity of study or work with obsessive determination, ignoring every other interest. Even if the beginning can be similar to innocuous dietetic slimming regime, what distinguishes anorexic patient by the contemporary to diet, are the tenacity with which the objective of to make lose weight is followed and the absolute rigidity by the dietetic regime. The meal begin early to becomes early a motive of anxiety so the patient tries to delay as much as possible the moment to sit down at table, he sometimes tills to subvert completely the hours, postponing all activities [20-30].

Many patients at the alimentary restriction associate physical activity whose very soon start increasing frequency and duration and they rapidly assume a compulsive modality.

In the phase of disorders, the worry for the weight assume obsessive characteristics, of predominant idea or even of delirious conviction. The amenorrhea appears very precociously, sometimes after that the loss of weight results evident. The latent hunger, even if denied, resurfaces both disguised as bulimic crisis, both by a constant presence of food at the centre of patient's. With advance of disorders, alimentation, practice of control of weight, styles of life, become more and more severe and stereotyped. In spite of the serious psychophysical poor state of health, is unaltered the terror to become stout and the conviction to be in overweight for all the skinny aspect. At the objective examination appear signs and symptoms by the extreme malnutrition: patients lament constipation and abdominal algia, skin appears dies, dehydrate, with typical yellow coloration and appears both lanugo that alopecia [20-30].

ECG and EEG are modified as regards norm. Patients are hypotensive and bradycardie; hemato-chemical tests show alteration of the hematic crasis and the hepatic and renal functions.
In the anorexic patients any weight loss seem to be sufficient: also when are seriously underweight they continue to feel too much fat or to feel some part of body like too much fat. The distortion by bodily image is the psychopathological nuclear aspect more resistant in each attempt of confutation and in many patients it assumes the aspect of the delirium [20-30].

The course of AN is variable: it can be characterized by the remission more or less complete, in consequence of single episode, particularly in more young patients and with better pre-existing social or working adaptation. In the $50 \%$ of cases well remain residual symptoms or psychopathological sequence, like the presence of depressive symptoms or dependence by drugs. The mortality varies between $5-20 \%$. Even if the normalization of the weight can be left anomaly of the alimentary pattern and the relation with the food can remain altered for a long time, with caloric restrictions, constant worry for the weight and bulimia [20-30].

\section{Bulimia nervosa}

Bulimia means "hunger of ox", "voracious appetite", "pathological voracity for the food" and intends the taking of big quantity of food in short time and with extreme voracity. This term was introduced in medicine for the first time by Gerard Russel in the 1979 [20-30].

It's a disease of the eating disorders characterized by episodes of rapid ingestion of large quantity of food preferably with high caloric contents (by 1000 to 20000 kilocalories); these episodes generally happen in solitude and are accompanied by a sensation of incapacity to control impulses, sense of guilt and psychic depression. These episodes generally last less of two hours and, in most cases, patients report a daily or pluri-daily frequency of this crisis. The bulimic episodes are made worse by unpleasant mood like sentiment of solitude and boredom, sadness, anxiety, anger, irritation, but often is the view of forbidden foods to provoke crisis [20-30].

The food choose to start the bingeing is generally one of which the patient tends to avoid in the one's dietetic scheme or that however he consider dangerous. The ingestion is confused, voracious, compulsive, accompanied by the sensation of loss of control. The patients feel, often, the sensation of not to be able to give up of eat, until no intervention outside cause at their will, like pain, sleep, intervention of third, deletion of food. Many patients report to calm their anxiety with the sensation of fullness and of abdominal tension, others of vomiting and then to perceive the sensation of relief deriving by to feel emptied. Frequently the thought of bingeing becomes dominant and it seriously interferes with the execution of daily activities, compromising the elaborate activities and the interpersonal relationships [20-30].

By a biological point of view the more reliable hypothesis is that bulimic behaviours can be considered a mechanism act to compensate the dysfunctional of the serotonergic central system; the serotonin is strictly involved in the genesis of the satiety signals. The bulimic crises is, in theory, compatible with the hypothesis of the reduction of serotonergic tone since bulimic patients show a net reduction of satiety sense and increase of hunger. We can assume that the bingeing serve to increase the synthesis of serotonin (that was reduced by an hypocaloric regime) compensating like this a reduced serotonergic tone. These binge eating induce some unsuited compensatory behaviours, put into action to avoid the weight increase like vomiting self-induced (sign of Russel on the back of the hand), abuse of laxatives or diuretics, clysters or other drugs [20-30]. 
Anorexic patients as well as bulimic patients frequently perform physical exercises to counterbalance the bingeing. In many cases, this compensatory behaviour become obsessive and compulsive. Unlike the anorexic patients, bulimic patient generally have a greater level of "insight" in other words they realize that the "war of food" coerce their life and their during interpersonal relationship [20-30].

This pathology usually start in adolescence or in first flush of youth, with a middle age of 18 years. Frequently the beginning appears afterwards a period of dietetic restriction. The functional compromise is variable, there are form well compensated and forms with a marked trouble of social function. Generally, also after the remission of bulimic crises persist a ideational polarization on food, weight and physical aspect and an highest risk of psychiatric disease: these subsyndromic forms be left in the $30 \%$ of cases. The disease become chronic in the $20 \%$ of patients. The mortality rate, associated at $\mathrm{BN}$, is not precise but can be about 5-7\% [20-30].

Many patients, even if vomit more than once a day, maintain a normal weight respect at their age and at their height but there are patients with a weight below norm and patients lightly obese.

Often it was described that bulimic patients show a poor control of impulses and often they present in comorbidity: kleptomania, tricotylomania, pathological gambling, confused sexuality and selfinjury impulses like scratch, cut, bit, fight oneself, ext. Also if rarely, it is also possible, that there are verified attempt of suicide, the most of times they are put in act with impulsive modalities and not programmed [20-30].

The BN implies serious risks also on the somatic system, some are more serious, like the gastric breaking or the oesophageal laceration. Frequent are also the hydro-electrolytic imbalance secondary to the vomit that can cause the cardiac arrhythmia or sudden death [20-30].

\section{Binge eating disorders}

The partial syndromes, called also sub-clinical or subliminal, are characterized by the presence of some, but not all, the symptoms necessary for the diagnosis of $\mathrm{AN}$ or BN. In the last years, between the various partial syndrome, provoked a particular interest, was provoked also for the high frequency by a clinical picture called in English "Binge Eating Disorders" (BED). In this syndrome are present crises of compulsive greed, of irresistible avidity for food but the serious and dangerous purging behaviours of the $\mathrm{BN}$ are not present [20-30].

The expression binge eating was not invented by a doctor but by a patient, the thirty-seven years old teacher of "Liceo H. Cohen" in treatment for obesity with Albert Stunkard toward the end of years '50. Binge eating derives, for analogy by binge dinking, to drink a bit too much, to lose measure of drinking. Literally it means: give at temptation to eat, to be indulgent with their voracity; to commit sin of gluttony. In reality Mr. Choen was a compulsive heavy eater and he defined the binge eating a particular type of transgression to the diet prescribed; indicating the moment when gave at temptation to eat without restraint, without can resist. No simple sin of gluttony, then, but occasion where the patient lost the control and, this it is a detail of big psychopathological interest, his state of conscience was altered. In synthesis the expression binge eating is used, today, in medicine and in psychiatry both to define a symptom, precisely the binge eating transversally passes through entire area of ED and all the spectrum of body weights, that for to indicate the name of the BED syndrome. It's necessary to underline the importance of BED for the high frequency of obese patients affect by BED. In fact the BED is relatively rare in the general population, with a percentage of prevalence included between
0,7 and $4,6 \%$, while it is commonly verified in obese patients, with value prevalently increasing side by side at level of BMI (between 30 and $70 \%)$. On the contrary than verified in $\mathrm{AN}$ and $\mathrm{BN}$, the BED can be more frequent in the female $(3: 2)$.

The psychopathological nucleus in BED such as in the BN is constituted by presence of binge eating. In the BED it should more correctly to speak about days instead of episodes of binge. An uncontrolled diet is in fact characterized more that by single bulimic crisis more or less frequent, by day of binge in which the patient ingests vast quantity of food alternated at day of correct diet or characterized by alimentary restriction [20-30].

Besides binge eating and alimentary restriction appear tightly connected through two important components: the frequency to the dis-inhibition and the susceptibility of the eat. In fact patients affected by BED present frequently a continuous struggle to avoid the binge eating and the aspiration to elevated standard of perfection to search to follow a dietetic and slimming regime.

The organic complications bound to BED are super-imposable to these of the simple obesity (diabetes mellitus, hypertension, cardiovascular diseases, some type of neoplasia, osteoarticular diseases, ext.) and their impact enlarge side by side to level of obesity [20-30].

It's commonly verified in the BED patients a presence of comorbidity, current or previous, with other psychiatric disorders, with percentage of psychopathologic superior to these of obese patients but inferior to these of bulimic patients. However we have verified in the BED patients a greater prevalence lifetime of depression, dysthymia, and borderline personality disorders.

\section{Control of appetite and weight-Role of neurochemical and neuropeptides systems}

The behaviours of search and ingestion of food or Alimentary Conducts (AC) can be interpreted as a final way resultant by the integration of exogenous and endogenous signals. The AC often shows quantitative or qualitative modifications in function of several environmental variables like ethnic- cultural, religious, climatic; but also inside of the same individual, there are alteration of AC that show clearly how is deep-rooted his adaptation homeostatic value, directed to preservation of the constancy of internal means in site variation of environmental conditions. The neurobiological research uses an integrated interdisciplinary approach to obtain information on determinants of ED [31-40]. The eating behaviour is regulated by several factors origin both exogenouses and endogenouses. The different informations coming both by internal and by external find their integration to hypothalamic level, also if today we prefer to speak about hypothalamus like centre of "satiety" and of "hunger" $(5,6)$. Clearly, besides this central function of hypothalamus there are in the Central Nervous System (CNS) also other specialized areas. Between these areas there is: (inferior tract of encephalic trunk and, particularly, the dorsal vagal complex) that receives and integrates the information that arrive by periphery autonomous endocrine organs and by different cerebral areas. Neuronal circuits of the mesencephalic trunk and of the thalamus interpret these information in relation to signals generated by the mechanical property of foods, that are obtained a different level of the gastrointestinal system [31-40]. The nucleus accumbens, the amygdale and the frontal cortex are responsible, instead, of more important functions that implicate the integration of cognitive information that regard the sensation of pleasantness or adversity to food. At the hypothalamic level, in virtue of its intense vascularization 
and of nervous projections by the inferior tract of the encephalic trunk, it happens an exact monitoring of the haematic levels of nutriments, of hormones and of the signal that arrive by the periphery. All these information have a deep impact on the activities of the neurochemical and neuroendocrine systems of hypothalamus that, in its turn, transfers signals act to influence the behavioural and metabolic processes [31-40]. All these neuronal systems, coordinating signals of several neurotransmitters and hormones as the amino acids, amines, peptides, and other steroidal hormones, are implicate in the control process of ingestion of food and of energy balance and so of the body weight. Naturally, these fine and precise neurochemical balances can be altered also by drugs that act on these modulators causing, in this way, alteration of the ED and consequently of the body weight. The control of ingestion of food results by the prevalence of factors to promoter action (neuropeptide $\mathrm{Y}$, nor-epinephrine, GABA, opioid peptides) by inhibitory factors (serotonin, CRF, prostaglandin) that interact as a model to cascade. On this central system enters a peripheral system responsible to send of hormonal and nervous signals coming by the gastro enteric tract, by metabolic signals by adipose tissue and other generated by nutriments present in circulation. This model is made more complex by the modulating effect explained, particularly, by circulating levels of the some hormones like insulin, steroids, and thyroids hormones [31-40].

The neuronal circuits that controls the ingestion of food is mainly related to catecholaminergic, serotonergic signals and peptidergic systems; also opioids have a really important role in the control of appetite, but certainly an essential role in this complex mechanism is done by the neuropeptides that have an inhibitory or stimulating action and can act by they self or in synergy with other neuromodulators [31-40].

Regarding the catecholamines, it has been observed that both noradrenaline (NA) and adrenaline (A) at the level of paraventricular nucleus of hypothalamus (PVN) stimulate the ingestion of food. By contrast, A,NA and dopamine (D) exert an anorexic action when they act at level of adrenergic and dopaminergic receptors in the perifornical area of lateral hypothalamus [31-40]. The role of serotonergic system at the level of PVN and of arcuate nucleus (ARC) of hypothalamus is to produce satiety. From data we know how factors that act, stimulating or inhibiting, on these system of neurotransmission, are able to interfere on the regulation of the bringing of food, on the structure of meal and on the choice of nutriments. Apart from the neurotransmitters exist numerous peptides able to do inhibitory or stimulatory activities that can act with a central or peripheral mechanism [31-40]. The anatomic seat of action of the neuropeptides at the central level is the same neuron modulating its action. The more effective stimulatory of appetite is certainly the neuropeptide Y (NPY); also the galanine and the opioid peptides are stimulatory of the ingestion of food. Besides peptides are able to determine the preference of certain nutriments, that is carbohydrates (NPY), carbohydrates and lipids (oppioides); lipids (galanine). Other peptides like the cholecystokinin (CCK), the bombesin (BBS), the somatostatine, the glucagons and other, belong to a complex peripheral system of the satiety, that in part carries on its action thanks to the use of numerous fibre coming by the vagus. At the peripheral level, and particularly in the gastro enteric tract, are secreted the PYY (Peptide YY) and PP (Peptide P); these molecules, similar for structure to NPY, are potent stimulatory of the appetite if injected to the hypothalamus. Therefore the gastro enteric tract through the various stimulus conduct by vagus nerve, has certainly a relevant role in the regulation of the diet [31-40].

So we must to remember that recently is been cloned in the adipose tissue of mouse and in the man one the gene OB that codifies for the protein leptine, which reduces the ingestion of food and the weight increase with an action at hypothalamic level acting on the sensation of hunger, the energetic consumption and body temperature.

\section{Adrenaline and noradrenaline}

Adrenalin and Noradrenalin control the adiposity and the energetic balance through several mechanisms: they promote the catabolism of triglycerides and glycogen, stimulate the ingestion of food when are injected in the CSN, activate the thermo genesis in the brown tissue and regulate the loss of calories through the modulation of the peripheral vasoconstriction. The stimulation aside of NA of a2-adrenergic hypothalamic receptors induces the ingestion of food through the increase of the quantity and duration of foods rich in carbohydrates. These response are anatomically localized at the level of paraventricular nucleus (PVN). In the perifornical area of lateral hypothalamus ( $\mathrm{LH})$, the NA and A, through the stimulation of $\beta$-adrenergic receptors, induce the suppression of ingestion of food delaying the beginning of meal, reducing the time spent to eat and the quantity of food introduced for meal. The amphetamine and similar, that induce a release both of NA that of dopamine, cause a strong stimulation of $\beta$-adrenergic and dopaminergic receptors. This kind of stimulation induces a reduction in particular in the ingestion of proteins [31-45].

\section{Dopamine}

The dopamine is able to suppress the ingestion of food through the activation of central dopaminergic receptors. In fact the systemic administration of dopaminergic agonists causes the reduction of the quantity of food ingested. As D as the agents that induce release of D reduce in particular the ingestion of foods rich in proteins and lipids, while have minor effect on the consumption of carbohydrates, the $\mathrm{D}$ attenuates the effect of galanine and of opiate on the ingestion of lipids [31-45].

The block of dopaminergic receptors in the lateral hypothalamus, through the injection of neuroleptic antagonist of dopaminergic receptors, provokes the opposite effects and, in particular an increased of consumption of proteins and lipids and so weight increase.

At last, we must to remember that recently is has been established a significant statistically correlation between the presence of a particular allele of $\mathrm{D}_{2}$ receptors and some aspects of eating behaviours in obese patients [31-45].

\section{Serotonin (5-HT)}

Substances that increase the activity of the serotonergic system induce an immediate and prominent reduction of ingestion of food, while the serotonergic antagonists cause an increase of it. The serotonin reduces the ingestion of food interacting with 5 -HT1b post-synaptic receptors that are concentrated in the medial hypothalamic nucleus and that are sensitive to the deprivation of food. The hypothalamic administration of 5-HT induces a pattern of ingestion of food contrary to that observed after $\alpha 2$-adrenergic stimulation. In fact we can note a reduction of the percentage of carbohydrates ingest respect to the proteins. We can see a similar pattern of taking of macronutrients after injection of fluoxetine in PVN, that can stop the reuptake of 5-HT from pre-synaptic endings. The serotonin and the drugs that induce the release of it, reduce the quantity and duration of meal, while they have a low effect on number of consumed meal [31-45].

Besides is necessary remember that a reduced taking of food could alter the hypothalamic serotonergic activity: the diet particularly reduces the concentration of tryptophan, amino acid precursory of 5-HT. This reduce the level of cerebral tryptophan, reducing the 
synthesis of serotonin. So the reduced taking inhibiting the synthesis of 5HT hypothalamic could induce a compensatory up-regulation in the response of 5-HT2C receptors. Then the extreme dieting provoke a reduced signal of satiety induced by the same serotonin.

We know that repeated and prolonged diets are the most important causes of the beginning of eating disorders, like the bulimia nervosa, that is a state characterized by episodes of binge eating [31-45].

\section{Gamma-aminobutyric acid (GABA)}

The gamma-aminobutyric acid, if injected in the PVN, develops the consumption of food and, particularly that one rich of carbohydrates. The GABA carries on its action through the activation of GABA receptors. Probably, this amino acid operates by coordinator between metabolic and neuronal signals that modulate the taking of the carbohydrates. At last, has been showed that the benzodiazepine, agonists of receptors for the GABA, provoke an increase of ingestion of food [31-45].

\section{Neuropeptide Y (NPY)}

The neuropeptide $\mathrm{Y}$ is a polypeptides of 36 amino acids that belongs to the pancreatic polypeptides' family and was been isolated for the first time in the 1982. It is considered the most potent stimulator of appetite (10). It is situated in different zones of brain. It is stays in elevated concentrations in the cortex and in corpus striated, in structures limbic, in the adrenergic and noradrenergic neurons that project versus the hypothalamus and in neuron one's of hypothalamus. The NPY to perform its action must legate to specific receptors: Y1, Y2 and $\mathrm{Y} 3$. The SNC contains $\mathrm{Y} 1$ and $\mathrm{Y} 2$ receptors. The action of stimulate on the appetite is followed by the activation of Y1 receptor [31-45].

\section{Galanin}

The galanin is a polypeptide made up of 29 amino acids and it is amply distributed in everywhere of gastro enteric tract and in brain. An injection of galanin in PVN produce in way dose-dependent, an increase of taking of food. The galanin operates through $a 2$-adrenergic receptors [31-45].

\section{Opioids}

Since a long time we know that the opioids stimulate the appetite and that an injection intracerebroventricular of $\beta$-endorfin increases the appetite but in a less marked way respect to NPY. Probably, opioids have a role also in process that regulate the use of energy interfering in particular on the thermo genetic processes. This data derives by the observation of patients who are in treatment with metadone: they are in fact most sub-weight, even if they preserving an caloric introit equal or also superior to the necessary for the preservation of ideal weight to cause of activation of processes of energetic dispersion [31-45].

\section{Treatment of Anorexia nervosa}

The first description of two probable cases of anorexia go back to 1689 when Richard Morton, in England, spoke of phthisis nervosa. He highlighted all the symptoms of current forms but without the worry to put on a lot weight. Two century in the 1868, W. Gull, described a patient with similar symptomatology, and he propose to call it hysteric apepsia. Some years later, in the 1873, Gull spoke more diffusely about a case with the same symptomatology but he preferred to call it "anorexia nervosa" and instead of hysteric, to underline the psychological component $[6,12,15]$.
Both Morton and Gull, later, raised questions on the therapy still present today, that is:

- The empiric character of the prescription;

- The large use of symptomatical, the use, with or after, of drugs and moral indications relative to the diet and to the style of life;

- Uncertainly of results and of outcome a long term;

- The refusal of treatment or, most precisely, of recovery.

They are problems with which still today we must deal.

Still the second middle of twentieth century, the psychopharmacology has done large progresses, by the synthesis, in the 1952 of the progenitor of the neuroleptics, the chlorpromazine, till neuroleptics of latest generation, the so called atypical and to the SSRI ext. Is important to underline that a large part of psychoactive drugs, gradually discovered had been tested in the treatment of ED $[6,12,15]$.

But, in spite of much general progresses of the psychopharmacology, between the drugs identified as useful in the treatment of $\mathrm{AN}, \mathrm{BN}$ and obesity, anyone has characters of wonder drugs, expression that means, the beginning of a drug of new, stupefying efficacy $[6,12,15]$.

In fact the data in actual literature don't show the real efficacy of any drugs in this group of diseases, unless for limited time and only on some symptoms. The psychological treatments are, for now, the cornerstone of the therapy of these diseases. The same dietetic prescription, could be defined as a psychotherapeutic intervention not formalized:

- Psychotherapeutic, because it tries to modify with psychical means (information, prescription, persuasion, ext) behaviours, complex personal and familiar equilibrium;

- No formalized because, generally, ii is not preceded by a precise psychological valuation and is not inspired by a theory or guided by a technique. Probably this defect is one of the most important causes of the failure collected by the diet-therapy. So drugs have a rule still limited, not decisive and not sufficient. But they is an aid more precious and more studies have demonstrated their utility. In the ED is field, necessary, as in some others fields of psychiatry and general medicine, to combine, case for case, the better pharmacological therapy possible with the appropriate case psychotherapeutic treatment, designing, from time to time, the strategy of intervention in function of the kind of pathology (indicator of diagnosis) and of the individual characteristics of single patient (clinics indicator)

Follow now a summary of drugs more used and more useful in the treatment of ED.

\section{Antipsychotic}

These drugs have represented, on empiric basis, the first systematic attempt of psychopharmacological intervention in AN. The rational of their used is bound to the hypothesis of a dopaminergic dysfunction in AN. Recent studies have valued that the use of the clozapine (antagonist D1/D4, D2/D3, agonist 5-HT2A/2C and 5-HT3) is potentially useful but, the tolerability and the necessity of frequent haematic check given, its use is an exception. Maybe the olanzapine (antagonist D1/D2/D4/5HT2C) have more possibility of use for its grater handiness. However, according to some recent met-analysis, in AN's treatment, the use of antipsychotic drugs, hasn't induced significant benefit in the ED, of weight, of hyperactivity and, unfortunately, neither in the area of illness of body image $[6,12,15]$. 


\section{Tricyclic Antidepressant (TC)}

In the clinical practice, the antidepressant tricyclic with SSRI, are the psycho drugs most frequently used in the AN treatment. The target symptoms are the depressive manifestations and the anxiety with recover of weight. Their use respond to the hypothesis of disfunction of monoamine $[6,12,15]$.

\section{Cyproheptadine}

It is the central agonist of serotonin and histamine, it acts reducing the central serotonergic activity and stimulates the ingestion of food. It possesses also sedative effects. The results of some studies have showed a better response in cases of restrictive anorexia, but it not exist evidence of the efficacy such to induce its use in the clinical practice $[6,12,15]$.

\section{Selective Serotonin Reuptake Inhibitors (SSRI)}

This drugs are been used in first place for the aspect of comorbidity, as modulator of humour and control of impulses. Their use responds to hypothesis of a serotonergic dysfunction in the AN. The SSRI act blocking the reuptake of serotonin in the synapses. The deficit of tryptophan (precursory of serotonin) caused by the malnutrition could make these drugs less efficacy, consequently hey must be given with an adequate nutritional counselling. Their use is supported by the elevated tolerability as regards antidepressant tricyclic with a greater or equal efficacy. Between the various SSRI the fluoxetine seems to be the molecule that has produced the most encouraging results. Some open studies have underlined that the fluoxetine seems to be able to promote the preservation in the time of the weight increase obtained during the acute phase of treatment and, then, to prevent the relapses. But remain some doubts about dosage, moment of prescription and total duration of therapy. Also the citalopram, that has a profile of more tolerability as regards to antidepressant tricyclic and also respect to others SSRI, has showed a specific effect on the increase of the appetite $[6,12,15]$.

\section{Lithium}

The salts of lithium are used in AN as mood regulating and the common side effect that produce is the weight increase. These are only the anecdotal descriptions without evidence of efficacy $[6,12,15]$.

\section{Anxiolytics}

The anxiety is a frequent symptoms in AN. It is associated in particular to the act of eat. Anyhow, the use of minor tranquilizer in AN must be occasional and limited in the time, because there are not evidences to the systematic use $[6,12,15]$.

Is important to underline, then, that anorexic patients live the pharmacological intervention as an invasive and uncontrollable action, like a strong, toxic and aggressive treatment to witch patients oppose their self. The resistance to start the treatment is one of the pathologic characteristics more frequent in AN. A drug orally administered sometime represents a foreign body that introducing himself in the organism can have immediate and profound effects not only on the weight and figure but also on the character and liberty and, like the food, it is refused or vomited. The therapy of AN consists to impose concurrent or sequential interventions, of individual and/or familiar, nutritional and pharmacological psychotherapy, since the symptoms of diseases are multi-determinate by biological, psychological and familiar factors. The pharmacological therapy is inserted in a polyphonic programme and it never is a single intervention. It must to be supported to the psychotherapy and to nutritional counselling. The first one will consider reasons of the treatment, resistance to the changer, of the importance of symptoms and, in particular, of the importance that have drugs on the patient is imaginary. The second one will give the necessary nutritious to the survival and functioning of drugs, but, without, promoting a sudden weight increase, cause frequently the interruption of treatment, also pharmacological $[6,12,15]$.

\section{Treatment of Bulimia nervosa}

Neurochemical, endocrinological and comorbidity studies have conduced to involve a reduction of the serotonergic transmission in the etiopathogenesis of the BN.

\section{Selective Serotonin Reuptake Inhibitors (SSRI)}

In this moment the fluoxetine is the only drug that have the specific indication for the treatment of $\mathrm{BN}$. several studies have showed that at dose of $60 \mathrm{mg}$ die is able to induce a significant reduction of the frequency of the bingeing and self-induce vomiting (17). At the reduction of the bulimic behavioural is associated an improvement of the depressive symptomatology and of the impulsiveness and a minimization of the craving for carbohydrates. Other SSRI like fluvoxamine, sertraline, citalopram, even if they not posses a specific indication can be used, usefully, in the treatment of $\mathrm{BN}[6,12,15]$.

\section{Tricyclic Antidepressant (TC)}

Studies both in open that in blind have highlighted the efficacy of antidepressant tricyclic in the treatment of BN. But in this moment the TC are a treatment of second rate for the better manageability and tolerability of SSRI associated to a same efficacy $[6,12,15]$.

\section{Mood stabilizers}

Some authors have suggested that the use of salts of lithium, in a little group of patients, is been able to reduce the number of binge crises. The reduced therapeutic window, the risk of secondary hydro electrical to vomit and the use of laxative and also the effect on the body weight do not advise their use $[6,12,15]$.

Recently some mood stabilizers like the gabapentin and the topiramate are been proposed in the treatment on $\mathrm{BN}$ because, particularly the topiramate, have highlight a marked anorexic effect. But at moment there are not controlled studies of efficacy.

The treatment in short term seems to be able to reduce about $70 \%$ the frequency of bingeing and purging behaviours, besides to improve the emotional component. More uncertain is the effect to long term of the pharmacological therapy. Follow-up is studies have highlighted like the suspension of drug is, generally, followed by a rapid relapse of the bulimic symptomatology, of the emotional lability and of increment of sense of hunger $[6,12,15]$.

\section{Treatment of binge eating disorders}

\section{Selective Serotonin Reuptake Inhibitors (SSRI)}

The use of fluoxetine in obese patients with BED, at the doses of 60 $\mathrm{mg}$, seems to have a positive effect on the reduction of weight and in part reduces the number of binge attacks. It seems also to improve the glucidic profile in obese patients affected by diabetes mellitus of type II but not secondary to the loss of weight, but it seems mediated by an increase of the synthesis of glycogen in the skeletric muscles $[6,12,15]$.

Also the fluvoxamine, at doses included between 100 and $200 \mathrm{mg}$ die and the sertraline with doses included between 150 and $200 \mathrm{mg}$ die seem to be able to reduce the frequency of the bingeing, the quantity 
of food and the fraction of carbohydrate, apart form to facilitate the induction of satiety $[6,12,15]$.

\section{Serotonin and Noradrenalin Reuptake Inhibitors (SNRI)}

The sibutramine through a mechanism of inhibition of reuptake of serotonin and noradrenalin promote a development of sense of satiety and a stimulation, even if minimum, of the thermogenesis. Through this mechanisms it causes a loss of weight and is associated a reduction of triglyceride, cholesterol LDL and increase of cholesterol HDL. At the moment are in being studies to verify if this drugs has the capacity, like postulate by its mechanism of action of reduce also the binge behaviours $[6,12,15]$.

The treatment of BED imply the difficulty implied to treat at the same time an eating disorders and a condition of obesity present in the most of these patients. Even though the overweight is only the consequence more evident, the symptomatologic nucleus is constituted by the bingeing but the loss of weight represent a necessary stage in the control of this disease.

The data present in literature show like the antidepressant drugs exert a positive effect a short term in to reduce the frequency of binge. Regard to the evolution to long term a study of follow-up to 3 and 6 years has showed an essential improvement of the symptomatology during the treatment a light re-accentuation during the first 3 years by the suspension of the treatment, followed by an ulterior improvement and stabilization by the fourth to sixth years $[6,12,15]$.

In the BED is indicated a therapeutic strategy where at the use, in initial phase of the pharmacological therapy for to begin the loss of weight, follows an interpersonal cognitive-behavioural psychotherapy, directed to act on psychological factors implicated in the etiopathogenesis of this disorders $[6,12,15]$.

\section{Conclusions}

In conclusion, in these last years the knowledge in the physiopathologist field of eating disorders have increased and is probable that this will consent, in the distance future, to discover molecules most sure and aimed to the pharmacological treatment of ED and of the weight. The studies on leptin, de-coupled proteins, $\beta 3$ adrenergic agonists, SSRI, are rich of expectations.

"Safer and better targeted medications for the drugs treatments of obesity and eating disorders can now be designed" Albert Stunkard 2000.

But the only biological evidences appear still distant to explain the complexity of AN, BN, BED and obesity. We know still little about integration between base's processes and superior mental functions in ED that we had to consider, for various aspects, a psychopathology. We think, for example, to communicative and social elements of the alimentation, at symbolic implications, at connections with the psychopathology of body's image, enjoyment, anxiety, wrong, shame, taste and disgust that modulate the report between man and food.

Waiting the wonder drug, we don't remain that continue to work, in a way most refined and unprejudiced, on the comparison and dialogue between body's and psyche's science, through the methodological line that Edgard Morin call complex thought, loved by experts and to neuroscience studious.

\section{References}

1. Halmi KA (2013) Perplexities of treatment resistance in eating disorders. BMC Psychiatry 13: 292. [Crossref]
2. APA (2006) Practice guidelines for the treatment of patients with eating disorders. In Practice Guidelines for the Treatment of Psychiatric Disorders, 3rd edn (pp. 10971222). Arlington, VA: American Psychiatric Association.

3. Hay P, Chinn D, Forbes D, Madden S, Newton R, et al. (2014) Royal Australian and New Zealand College of Psychiatrists clinical practice guidelines for the treatment of eating disorders. Aust N Z J Psychiatry 48: 977-1008. [Crossref]

4. Micali N, De Stavola B, Ploubidis G, Simonoff E, Treasure J, et al. (2015) Field Adolescent eating disorder behaviours and cognitions: gender-specific effects of child, maternal and family risk factors. Br J Psychiatry 207: 320-327. [Crossref]

5. Halmi KA (2009) Salient components of a comprehensive service for eating disorders World Psychiatry 8: 150-155. [Crossref]

6. Capasso A, Petrella C, Milano W (2009) Recent clinical aspects of eating disorders Rev Recent Clin Trials 4: 63-69. [Crossref]

7. Hudson JI, Hiripi E, Harrison G, Pope Jr. HG, Kessler RC (2007) The prevalence and correlates of eating disorders inthe national comorbidity survey replication. Biological Psychiatry 61: 348-358.

8. Keski-Rahkonen A, Mustelin L (2016) Epidemiology of eating disorders in Europe: prevalence, incidence, comorbidity, course, consequences, and risk factors. Curr Opin Psychiatry 29: 340-345.

9. Volpe U, Tortorella A, Manchia M, Monteleone AM, Albert U, et al. (2016) Eating disorders: What age at onset? Psychiatry Res 238: 225-227. [Crossref]

10. Chesney E, Goodwin GM, Fazel S (2014) Risks of all-cause and suicide mortality in mental disorders: a meta-review. World Psychiatry 13: 153-160. [Crossref]

11. Scanelli G, Gualandi M, Simoni M, Manzato E (2014) Somatic involvement assessed through a cumulative score of clinical severity in patients with eating disorders. Eat Weight Disord 19: 49-59. [Crossref]

12. Capasso A, Petrella C, Milano W (2009) Pharmacological Profile of SSRIs and SNRIs in the Treatment of Eating Disorders. Current Clinical Pharmacology 4: 78-83

13. Bulik C, Hebebrand J, Keski-Rahkonen A, Klump K, Reichborn-Kjennerud KS, et al (2007) Genetic epidemiology, endophenotypes, and eating disorder classification. Int J Eat Disord Suppl: S52-S60. [Crossref]

14. American Psychiatric Association (2013) Diagnostic and Statistical Manual of Mental Disorders. Fifth Edition. Washington, DC: American Psychiatric Association.

15. Milano W, De Rosa M, Milano L, Riccio A, Sanseverino B, et al. (2013) The pharmacological options in the treatment of eating disorders. ISRN Pharmacol 2013: 352865. [Crossref]

16. Culbert KM, Racine SE, Klump KL (2015) Research Review: What we have learned about the causes of eating disorders - a synthesis of sociocultural, psychological, and biological research. J Child Psychol Psychiatry 56: 1141-1164.

17. Treasure J, Claudino AM, Zucker N (2010) Eating disorders. Lancet 375: 583-593. [Crossref]

18. Easter MM (2012) "Not all my fault": Genetics, stigma, and personal responsibility for women with eating disorders. Soc Sci Med 75: 1408e1416. [Crossref]

19. Boraska V, Franklin CS, Floyd JA, Thornton LM, Huckins LM, et al. (2014) A genomewide association study of anorexia nervosa. Mol Psychiatry 19: 1085-1094. [Crossref]

20. Sharan P, Sundar AS (2015) Eating disorders in women. Indian J Psychiatry 57: S286295. [Crossref]

21. Val-Laillet D, Aarts E, Weber B, Ferrari M, Quaresima V, et al. (2015) Neuroimaging and neuromodulation approaches to study eating behavior and prevent and treat eating disorders and obesity. Neuroimage Clin 8: 1-31

22. Yilmaz Z, Hardaway JA, Bulik CM (2015) Genetics and Epigenetics of Eating Disorders. Adv Genomics Genet 5: 131-150. [Crossref]

23. Sim LA, McAlpine DE, Grothe KB, Himes SM, Cockerill RG, et al. (2010) Identification and Treatment of Eating Disorders in the Primary Care Setting. Mayo Clin Proc 85: 746-751. [Crossref]

24. Favaro A, Tenconi E, Santonastaso P (2006) Perinatal factors and the risk of developing anorexia nervosa and bulimia nervosa. Arch Gen Psychiatry 63: 82-88.

25. Petra AI, Panagiotidou S, Hatziagelaki E, Stewart JM, Conti P, et al. (2015) GutMicrobiota-Brain Axis and Its Effect on Neuropsychiatric Disorders With Suspected Immune Dysregulation. Clin Ther 37: 984-995. [Crossref]

26. Mu C, Yang Y, Zhu W (2016) Gut Microbiota: The Brain Peacekeeper. Front Microbiol 7: 345. [Crossref] 
27. Kaye W (2008) Neurobiology of anorexia and bulimia nervosa. Physiol Behav 94: 121135. [Crossref]

28. Roth J, Szulc AL, Danoff A (2011) Energy, evolution, and human diseases: an overview. Am J Clin Nutr 93: 875S-83. [Crossref]

29. Schwartz MW, Woods SC, Porte Jr D, Seeley RJ, Baskin DG (2000) Central nervous system control of food intake. Nature 404: 661-671.

30. Kaye W, Strober M, Jimerson D (2004) The neurobiology of eating disorders. In: Charney DS, Nestler EJ, editors. The neurobiology of mental illness. New York: Oxford Press pp: $1112-1128$.

31. Monteleone P, Castaldo E, Maj M (2008) Neuroendocrine dysregulation of food intake in eating disorders Regul Pept 149: 39-50. [Crossref]

32. Tong J, D'Alessio D (2011) Eating disorders and gastrointestinal peptides. Curr Opin Endocrinol Diabetes Obes 18: 42-49. [Crossref]

33. Hirschberg AL (2012) Sex hormones, appetite and eating behaviour in women. Maturitas 71: 248-256. [Crossref]

34. Tortorella A, Brambilla F, Fabrazzo M, Volpe U, Monteleone AM, et al. (2014) Central and Peripheral Peptides Regulating Eating Behaviour and Energy Homeostasis in Anorexia Nervosa and Bulimia Nervosa: A Literature Review. Eur Eat Disorders Rev 22: 307-320. [Crossref]

35. Cota D, Marsicano G, Lutz B, Vicennati V, Stalla GK, et al. (2003) Endogenous cannabinoid system as a modulator of food intake. Int J Obes Relat Metab Disord 27: 289-301.
36. Phillips ML, Drevets WC, Rauch SL, Lane R (2003) Neurobiology of emotion perception I: The neural basis of normal emotion perception. Biol Psychiatry 54: 504 514. [Crossref]

37. Kaye WH, Wagner A, Fudge JL, Paulus M (2011) Neurocircuity of Eating Disorders. Curr Topics Behav Neurosci 6: 37-57. [Crossref]

38. Monteleone P, Maj M (2013) Dysfunctions of leptin, ghrelin, BDNF and endocannabinoids in eating disorders: beyond the homeostatic control of food intake. Psychoneuroendocrinology 38: 312-330. [Crossref]

39. Monteleone AM, Di Marzo V, Monteleone P, Dalle Grave R, Aveta T, et al. (2016) Responses of peripheral endocannabinoids and endocannabinoid-related compounds to hedonic eating in obesity. Eur J Nutr 55: 1799-1805.

40. Culbert KM, Racine SE, Klump KL (2016) Hormonal Factors and Disturbances in Eating Disorders. Curr Psychiatry Rep 18: 65. [Crossref]

41. Cassin SE, von Ranson KM (2005) Personality and eating disorders: a decade in review. Clin Psychol Rev 25: 895-916. [Crossref]

42. Schwartz MW, Woods SC, Porte D Jr, Seeley RJ, Baskin DG (2000) Central nervous system control of food intake. Nature 404: 661-671. [Crossref]

43. Giuliano C, Cottone $\mathrm{P}$ (2015) The role of the opioid system in binge eating disorder. CNS Spectr 20: 537-545. [Crossref]

44. Spanagel R, Weiss F (1999) The dopamine hypothesis of reward: past and current status. Trends Neurosci 22: 521-527. [Crossref]

45. Hoebel BG (1985) Brain neurotransmitters in food and drug reward. Am J Clin Nutr 42 1133-1150. [Crossref]

Copyright: (C2017 Milano W. This is an open-access article distributed under the terms of the Creative Commons Attribution License, which permits unrestricted use, distribution, and reproduction in any medium, provided the original author and source are credited. 